\title{
Evaluación de la gestión de seguridad en la cadena de suministro en el sector ganadero de Panamá
}

\section{Evaluation of the security management in the supply chain in the livestock sector of Panamá}

\author{
Emily Saldaña ${ }^{1 *}$, Práxedes Torres ${ }^{2}$ \\ ${ }^{1}$ Licenciatura en Logística y Transporte Multimodal, Facultad de Ingeniería Industrial, Universidad Tecnológica de Panamá \\ ${ }^{2}$ Facultad de Ingeniería Industrial - Universidad Tecnológica de Panamá
}

\begin{abstract}
Resumen Las cadenas de suministro son vulnerables a varios riesgos que podrían romper la continuidad del negocio y hasta pueden hacer cerrar las operaciones. El sector ganadero de Panamá, base de las cadenas de suministro alimenticio, se ve afectado primordialmente por el hurto pecuario. La provincia de Chiriquí, provincia con mayor actividad ganadera del país, es la que presenta mayor afectación por este problema. El modelo de madurez de riesgo basado en la Norma ISO 31000:2009 se utilizó como herramienta para medir el nivel de implementación de la gestión de riesgo del sector ganadero resultando, en un nivel medianamente consistente.
\end{abstract}

Palabras clave Cadena de suministro, gestión de riesgos, hurto pecuario, modelo de madurez de riesgo, norma ISO 28000, norma ISO 31000 .

\begin{abstract}
Supply chains are vulnerable to a number of risks that could disrupt business continuity and can even shut down operations. Panama's livestock sector, the base of the food supply chains, is primarily affected by livestock theft. The province of Chiriquí, the province with the highest livestock activity in the country, is the one that is most affected by this problem. The risk maturity model based on the ISO 31000: 2009 standard was used as a tool to measure the level of implementation of risk management in the livestock sector, resulting in a fairly consistent level.
\end{abstract}

Keywords Supply chain, risk management, livestock theft, risk maturity model, standard ISO 28000, standard ISO 31000.

* Corresponding author: emilysaldana@utp.ac.pa

\section{Introducción}

Actualmente, las empresas se enfrentan a riesgos internos y externos que afectan directamente la continuidad del negocio, ejemplos de estos tenemos: inundaciones, incendios, vandalismo y robo. A estas se les incluye otros riesgos frecuentes, como el contrabando y los actos delictivos que interrumpen la cadena de suministro y la continuidad de la empresa.

En un mundo globalizado, donde el comercio está creciendo y se torna dinámico, es indispensable que las empresas realicen un estudio del riesgo e impacto de la cadena de suministro y sus posibles consecuencias al verse afectados por estos riesgos.

Como soporte a las organizaciones, se han desarrollado estándares internacionales basados en buenas prácticas para la gestión del riesgo ("security"). Normas como BASC y la ISO 28000 resaltan entre las más utilizadas para los temas de "security". Sin embargo, la Norma ISO 31000:2009 es una norma completa que sirve para cualquier tipo de organización que tiene planeado implementar la gestión de riesgo, tanto en el ámbito de seguridad operacional, como de seguridad física.

En este documento se muestra la investigación realizada consistente de la medición del nivel de implementación de la Norma ISO 31000:2009, que tiene como finalidad el proporcionar un marco de referencia para la gestión de un sistema global de la seguridad de la cadena logística y asî reducir la posibilidad que las operaciones sean utilizadas para ilícitos, ya que esta norma facilita a que las organizaciones se anticipen a dichos acontecimientos mediante la prevención.

La Norma ISO 31000 es una norma más genérica enfocada en la gestión del riesgo en cualquier tipo de organización. Su enfoque es, tanto en aspectos de seguridad operacional como de seguridad física. 
El instrumento utilizado para medir el nivel de implementación de una gestión de riesgo en una organización es por medio de modelos que midan el nivel de madurez de riesgo ("Risk Maturity Model" - RMM). Estos modelos sirven como indicadores que miden el cumplimiento de una serie de atributos que terminan en indicadores para medir el nivel alcanzado por la organización.

\section{Antecedentes}

Los sucesos terroristas ocurridos el 11 de septiembre de 2001, en Nueva York, Estados Unidos; entre ellos el más emblemático de dicho día, el dado en las Torres Gemelas, han traído como consecuencia que las organizaciones, tanto públicas como las del sector privado, tuvieran una mayor consciencia y control en cuanto a la necesidad de proteger sus bienes y activos, lo que ha propulsado a las diferentes entidades gestionar la creación de normas sobre la gestión de seguridad para la cadena de suministro [1].

Para proteger la seguridad de la cadena de suministro, Estados Unidos desarrolló el programa C-TPAT ("Customs Trade Partnership Against Terrorism" - Asociación Aduanera-Comercial contra el Terrorismo), el cual implementa medidas de seguridad que protege a la cadena de suministro, pues evita que esta sea utilizada por delincuentes y/o terroristas, como un camino fácil para realizar el delito. A su vez, la Organización Mundial de Aduanas, con el referente de "C-TPAT", estableció el Marco de Estándares de Seguridad (Framework of Standards), dando así la publicación de esta iniciativa en su Asamblea General del año 2005. De igual manera, el Parlamento Europeo, en ese mismo año introdujo una rectificación a su Código Aduanero Comunitario, permitiendo que las aduanas europeas otorguen beneficios a los responsables del comercio exterior para calificarse como Operador Económico Autorizado.

Como no existía un ente que enlazara ambas iniciativas, es donde entra la Organización Internacional de Normalización (ISO) y confecciona la Norma ISO 28000 en el 2007, en respuesta a las necesidades de las empresas y actualmente logra unir estándares diferentes a nivel mundial y alcanza a cerrar la brecha entre los programas OEA y C-TPAT, permitiendo así su reconocimiento mutuo [2].

Las certificaciones internacionales de procesos de calidad y seguridad como: ISO, BASC ("Business Alliance for Secure Commerce" - Alianza Empresarial Internacional), TAPA ("Transported Asset Protection Association" - Asociación para la protección de activos transportados), OEA, C-TPAT son exigidos mundialmente como requisito inicial de muchos clientes. Los dos únicos sistemas de gestión en el mundo para asegurar cadenas de suministro son: Norma ISO 28000 y BASC; los demás son iniciativas de seguridad que cuentan con requisitos de este, mas no cumplen con las características de un sistema de gestión [3].

En Panamá, hemos tenido casos de rompimiento de la cadena de suministro como, por ejemplo, la Autoridad Nacional de Aduanas (ANA), en el mes de diciembre de 2018, declaró un paro laboral, en reclamo del pago de una bonificación, que se les adeudaba desde enero del mismo año [4]. Esto ocasionó afectaciones a los comercios debido a la retención de materia prima fundamental para la elaboración de productos. Una de las tantas empresas afectadas fue Krispy Kreme, que se vio obligada de cesar la operación en seis tiendas y restablecer la misma hasta que la carga saliera de aduana [5].

Posteriormente, la Asociación de Médicos Veterinarios, convocó a un paro de brazos caídos para el sector público, debido al incumplimiento de acuerdos salariales y el reconocimiento de los estudios de especialidades. Dicha medida tuvo afectación en las actividades de las diferentes entidades públicas: incluyendo los puertos, aeropuertos y puertos fronterizos. Este paro incluía la paralización de la entrada y salida de productos de origen animal, tanto por vía aérea, marítima y terrestre, al igual la paralización de los servicios en centros de salud, labores en las plantas de sacrificios y de proceso [6].

Es relevante que un país, al incrementar sus negociaciones también aumenta la inseguridad, ya que se puede presentar riesgos en el transporte, el almacenamiento de la mercancía, manipulación de esta y la presencia de personas ajenas que suelen aprovecharse de estas vulnerabilidades.

En países como México, Argentina y Uruguay, por sus características geográficas y sus actividades económicas, sufren de hurto pecuario. En Panamá, no se escapa a esta situación. Entre las mayores limitantes de este sector para su desempeño están: mercado saturado de productos importados en época de cosecha, falta de planificación y coordinación institucional efectiva en el sector gubernativo agropecuario, cambio climático, contrabando, falta de mano de obra y costos de producción [7].

Según informe del Ministerio Público del 2 de septiembre de 2016 al 31 de julio de 2017, se recibieron 651 denuncias por el delito contra el patrimonio económico, en su modalidad de hurto pecuario. Los datos estadísticos del Ministerio Público mencionan que las provincias que se reportó con más denuncias por este delito son: Chiriquí con 154; Los Santos, con 119 casos y Coclé, donde entraron 83 denuncias [8].

La mercancía es perecedera, en especial las cárnicas (aves, bovinos, equinos, entre otros). Para los ganaderos, el problema del hurto pecuario representa pérdidas monetarias, ya que el modus operandi de los abigeos/cuatreros es sustraer los animales de las fincas, luego llevarlas hasta el matadero, en 
donde los sacrifican y posteriormente terminar con su comercialización [9].

En la figura 1 se aprecia las denuncias registradas por hurto pecuario en las provincias de Chiriquí, Los Santos y Coclé.

DENUNCIAS REGISTRADAS POR HURTO PECUARIO

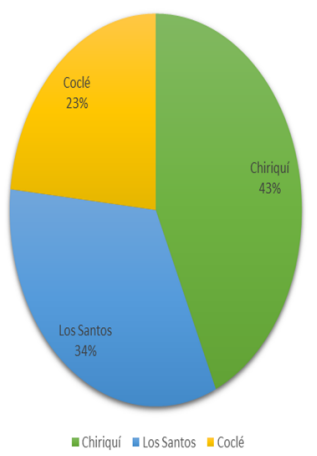

Figura 1. Denuncias por el delito contra el patrimonio económico, en su modalidad de hurto pecuario.

Para los fines de este trabajo se considera el sector ganadero de la provincia de Chiriquí, debido a la gran cantidad de denuncias registradas por la modalidad de hurto pecuario. Por ello, se quiso investigar cuál sería su nivel de madurez de su gestión de riesgo para afrontar los hurtos y los otros riesgos que esta industria afronta.

\section{Marco teórico}

\subsection{Sector ganadero}

Según el último censo agropecuario del año 2010, con respecto a la producción ganadera en Panamá, existen 10,897 explotaciones dedicadas a la ganadería bovina en la provincia de Chiriquí, de las cuales el $52 \%$ se dedica a la cría, el $26 \%$ a la ceba y el $22 \%$ restante a la producción lechera [10]. En la tabla 1 se puede observar la distribución de las fincas ganaderas y las reses según el tamaño de explotación en la provincia de Chiriquí.

Tabla 1. Distribución de las fincas ganaderas y las reses según el tamaño de explotación en la provincia de Chiriquí

\begin{tabular}{|c|c|c|}
\hline $\begin{array}{c}\text { Tamaño de la } \\
\text { finca }(\text { No. de } \\
\text { reses) }\end{array}$ & $\begin{array}{c}\text { Número de } \\
\text { fincas }\end{array}$ & $\begin{array}{c}\text { Número total de } \\
\text { animales }\end{array}$ \\
\hline $0-99$ & 6,150 & 173,577 \\
\hline $100-499$ & 496 & 114,728 \\
\hline $500-$ en adelante & 54 & 56,981 \\
\hline
\end{tabular}

Fuente: Contraloría General de la República de Panamá.

El proceso pecuario de producción de ganado en pie se clasifica en cinco etapas: Reproducción, cría, levante, Engorde y comercialización de ganado en pie [11].

\subsection{La importancia de la seguridad de la cadena de suministro}

La seguridad es un factor muy importante para el desarrollo de un comercio íntegro; esta se fortalece del aporte que empieza desde el proveedor hasta el vendedor. Sin embargo, existen otros factores que influyen en su consolidación o debilitamiento. Las empresas que no toman en serio la seguridad de la cadena de suministro se vuelven vulnerables a ataques potencialmente devastadores, por ejemplo: el terrorismo, la piratería y el robo [12].

La seguridad de la cadena de suministro busca la aplicación de políticas, procedimientos y tecnologías para velar por el correcto y oportuno funcionamiento de las cadenas de suministro [13].

\subsection{La Norma ISO 28000:2007}

La Norma ISO 28000:2007 es un estándar que permite a las empresas de cualquier sector, promover las mejores prácticas en auditoría de riesgos y manejo de eventos de seguridad en la cadena de suministro [14].

La Organización Internacional de Normalización (ISO), publicó en el año 2007 esta norma; la cual define requisitos y recomendaciones para aquellas empresas que están involucradas a la cadena de suministro.

Esta es la primera norma de este tipo, en señalar los riesgos que conlleva para la seguridad dentro de la cadena de suministro [15].

Esta norma fue revisada y confirmada por última vez en el año 2014 y sigue siendo esta versión actual. Para el año 2020 se espera su nueva versión [16].

La Norma ISO 28000 es una opción para que las empresas puedan establecer y documentar niveles razonables de seguridad, dentro de la cadena suministro y sus unidades. Esta norma es multimodal y no procura reemplazar o anular a los programas de seguridad de las cadenas de suministro de las direcciones de aduanas nacionales, es decir busca estar de acuerdo y complementar el Marco de Normas de la Organización Mundial de Aduanas para facilitar el comercio mundial [17].

Según la ISO, esta norma es aplicable a organizaciones de la pequeña, mediana y grande empresa, para las áreas de fabricación, servicio, almacenamiento o transporte; en cualquier etapa de la producción o cadena de suministro que desee establecer, implementar, mantener y mejorar un sistema de gestión de la seguridad, y a su vez, asegurar la conformidad con la política de gestión de seguridad establecida [18].

Esta norma requiere que la organización o empresa evalúe el entorno de seguridad en el que trabaja, determine si se ejecutan medidas de seguridad adecuadas y si ya existen otros requisitos reglamentarios que la misma cumpla. La base principal de la Norma ISO 28000 es la gestión del riesgo, desde 
un punto de vista de seguridad física - "security" como es conocido en inglés [19].

\subsubsection{Estructura de la Norma ISO 28000}

La estructura de la Norma ISO 28000 está compuesta por:

1. Política de gestión de la seguridad

2. Planificación y evaluación del riesgo de seguridad

- Evaluación de riesgos.

- Requerimientos legales, estatutarios y otros.

- Objetivos y metas de seguridad.

- Programas de Gestión de la Seguridad.

3. Implementación y operación

- Estructura, autoridad y responsabilidades.

- Competencia, formación y toma de conciencia.

- Comunicación.

- Documentación.

- Control de documentos.

- Control operacional.

- Preparación y respuesta ante emergencias y recuperación de la seguridad.

4. Verificación y acción correctiva

- Medición y monitoreo del desempeño de la seguridad.

- Evaluación del sistema.

- Fallas relacionadas con la seguridad, incidentes, no conformidades y acción correctiva y preventiva.

- Control de registros.

- Auditoría de S.G.S.

5. Revisión por la dirección y mejoramiento continuo.

6. Mejoramiento continuo [20].

\subsection{Diferencia entre "safety" y "security"}

En la lengua inglesa, seguridad es un término que tiene dos formas de mencionarlos: safety y security. Según Olleros la "Diferencias entre Safety y Security" es la siguiente: "Safety y Security son disciplinas que se ocupan de la protección y reducción de los riesgos de las personas y de su propiedad, contra los peligros y daños que tienen su origen en el delito, el uso indebido y en la accidentalidad de sistemas y procesos" [21].

Estos dos términos nos permiten diferenciar los riesgos de seguridad que enfrentan las empresas:

1. "Safety" (protección)/Seguridad operacional: Se refiere a todos los riesgos derivados de eventos accidentales o fortuitos como: desastres naturales, errores humanos, accidentes, fallos no intencionados, entre otros.

2. "Security" (seguridad)/Seguridad física: Son los riesgos en los cuales hay un oponente o atacante intencionado que provoca algún daño. Ejemplos: sabotajes, robos, fraudes, entre otros [22].

En la tabla 2 se describen las principales diferencias entre "Safety" y "Security".
Tabla 2. Principales diferencias entre "Safety" y "Security" [23]

\begin{tabular}{|c|c|c|}
\hline Características & "Safety" & "Security" \\
\hline Causas & $\begin{array}{l}\text { Comportamiento } \\
\text { humano en } \\
\text { combinación con } \\
\text { el ambiente. }\end{array}$ & $\begin{array}{l}\text { Incidente como } \\
\text { resultado de una } \\
\text { persona o grupo } \\
\text { de personas. }\end{array}$ \\
\hline Causas & $\begin{array}{l}\text { A menudo no } \\
\text { existen acciones } \\
\text { planificadas. }\end{array}$ & $\begin{array}{c}\text { A menudo } \\
\text { existen acciones } \\
\text { planificadas. }\end{array}$ \\
\hline Causas & $\begin{array}{l}\text { Actos delictivos } \\
\text { (Ley de Medio } \\
\text { Ambiente). }\end{array}$ & Actos delictivos. \\
\hline Causas & $\begin{array}{c}\text { Rara o } \\
\text { maliciosamente } \\
\text { ocurre. } \\
\end{array}$ & $\begin{array}{c}\text { Principalmente } \\
\text { ocurre actos } \\
\text { maliciosos. }\end{array}$ \\
\hline Causas & $\begin{array}{c}\text { Actos } \\
\text { deliberados sin } \\
\text { deseo de } \\
\text { ocasionar un } \\
\text { incidente. }\end{array}$ & $\begin{array}{c}\text { Actos } \\
\text { deliberados con } \\
\text { deseo de llevar } \\
\text { consigo una } \\
\text { consecuencia del } \\
\text { acto. }\end{array}$ \\
\hline Amenazas/peligros & $\begin{array}{c}\text { Amenazas } \\
\text { humanas } \\
\text { internas. }\end{array}$ & $\begin{array}{c}\text { Amenazas } \\
\text { humanas } \\
\text { internas y } \\
\text { externas. }\end{array}$ \\
\hline Amenazas/peligros & $\begin{array}{c}\text { Los peligros son } \\
\text { observables, } \\
\text { tangibles y } \\
\text { próximos }\end{array}$ & $\begin{array}{c}\text { Los peligros no } \\
\text { son observables, } \\
\text { tangibles y } \\
\text { próximos. }\end{array}$ \\
\hline Pérdidas & $\begin{array}{c}\text { Pérdida } \\
\text { relacionada con } \\
\text { lesiones } \\
\text { humanas, muerte } \\
\text { y fiabilidad de } \\
\text { activos } \\
\text { industriales. }\end{array}$ & $\begin{array}{l}\text { Pérdida } \\
\text { relacionada con } \\
\text { activos físicos e } \\
\text { información. }\end{array}$ \\
\hline Entorno & $\begin{array}{l}\text { Condiciones } \\
\text { físicas y } \\
\text { ambientales, no } \\
\text { solamente } \\
\text { humanos y la } \\
\text { sociedad. }\end{array}$ & $\begin{array}{l}\text { Estado de la } \\
\text { sociedad a } \\
\text { través de sus } \\
\text { estructuras, } \\
\text { situación } \\
\text { económica, } \\
\text { respecto a la ley } \\
\text { y moral. }\end{array}$ \\
\hline Relevancia & $\begin{array}{l}\text { La industria y el } \\
\text { sector transporte }\end{array}$ & $\begin{array}{c}\text { Amplia gama de } \\
\text { empresas. }\end{array}$ \\
\hline Incertidumbre & & $\begin{array}{l}\text { Alto grado de } \\
\text { incertidumbre y } \\
\text { bajo grado de } \\
\text { conocimiento } \\
\text { sobre amenazas. }\end{array}$ \\
\hline
\end{tabular}




\subsection{Norma ISO 31000:2009}

Para realizar una correcta identificación y evaluación de riesgos se necesita el apoyo en la Norma "ISO 31000 Principios y directrices para la gestión de riesgos" que establece que todo tipo de organizaciones independientemente de su tamaño y funciones, enfrentan factores e influencias, tanto internas y externas, que crean incertidumbre sobre los logros de sus objetivos. Es decir, todas las actividades que se desarrolla dentro de una organización implican riesgo.

Cada sector específico o cada aplicación de la gestión del riesgo lleva consigo requisitos, audiencias, evaluaciones y criterios individuales. Por lo tanto, es fundamental establecer el contexto de cada riesgo [19].

Las Normas ISO 31000, tienen un enfoque más general que las Normas ISO 28000, en cuanto a que la ISO 31000 ayuda a implementar procesos de gestión de riesgo en cualquier tipo de organización, por lo que toman en cuenta tanto las partes de seguridad operacional ("safety"), como de seguridad física ("security").

\subsection{El modelo de madurez de riesgo}

Los riesgos que ocurren dentro del sector ganadero se pueden deber a : caídas por suelo en malas condiciones, accidentes causados por los propios animales, contagio de diferentes enfermedades (tuberculosis bovina, brucelosis, hidatidosis, etc.) transmitidas por el ganado, accidentes fortuitos durante el manejo de los animales, falta de seguridad estructural en las construcciones, entre otros [24].

La Norma ISO 31000 (Sistemas de Gestión de Riesgos) cubre toda la gestión de la seguridad y al igual que toda norma ISO, esta se orienta a la mejora continua (Planificar, Realizar, Comprobar y Reaccionar). Es una excelente norma para la implementación de la gestión de seguridad en diversos tipos de empresas.

El grado de madurez en una empresa puede ser medido mediante modelos de madurez (RMM). Estos modelos permiten realizar una evaluación de los procesos de control, eliminación y mitigación de los sucesos que amenazan las operaciones de una empresa [25].

Dado que la investigación se enfocó en medir el nivel de implementación de la gestión de riesgo, asimismo se distingue que el sector productor ganadero tiene riesgos en ambas áreas: tanto en "safety" y "security". Es preferible utilizar el modelo de madurez de riesgo, el cual es un marco de referencia que sirve como indicador para reconocer el nivel de implementación de la gestión de riesgo de las organizaciones. Este indicador permite obtener un puntaje de madurez y pautas para mejorar sus programas ya establecidos. No hay un modelo único, ya que se pueden basar en diferentes criterios para la aplicación de esta.
El modelo de madurez de riesgos (RMM) permite a las organizaciones medir qué tan bien han realizado sus esfuerzos en implementar la gestión de riegos, "ya que es una herramienta de buenas prácticas para la gestión de riesgos empresariales" [26]. Este modelo tiene como resultado que las organizaciones reciban un puntaje de madurez y un conjunto de directrices para mejorar y obtener los muchos beneficios asociados con la madurez.

Por lo tanto, al ser seleccionada la Norma 31000, el instrumento a medir el nivel de implementación, nivel de madurez de riesgo, debe estar basado en la norma en mención.

Los modelos de madurez de riesgo en general tienen una estructura en la que se tienen una serie de atributos de las prácticas de gestión de riesgo y en la medida que cumpla con las buenas prácticas de gestión de riesgo, se clasifica la empresa en un nivel jerárquico que puede ser de 4 a 5 niveles generalmente.

\subsection{Análisis de los riesgos}

Toda empresa debe establecer procedimientos, cuyo principal objetivo sea su conservación. Este tipo de actividades ayudan a identificar y a evaluar las posibles amenazas de seguridad que puedan exhibirse dentro de la cadena de suministro, al igual que la toma de medidas de control indispensables para las diferentes escalas de operaciones.

La evaluación de riesgos incluye las amenazas y riesgos de fallo físico, desastres naturales, amenazas y riesgos naturales, amenazas y riesgos de partes afectadas, factores que no se pueda controlar dentro de la empresa, instalación de equipo de seguridad y amenazas al desarrollo de las operaciones [27].

Los riesgos más sobresalientes en el sector productor de ganado se dan primordialmente en temas de "security", dado por el hurto pecuario. Este acto delictivo consiste en el robo del ganado de cualquier tipo: bovino, equino, entre otros, de las fincas. Sus modalidades se listan:

- Atraco: Es cuando el robo surge a través de la intimidación. En este delito puede ocurrir en el secuestro, donde los personajes que se roban el ganado cometen el acto, sus cómplices amordazan y atemorizan a sus víctimas.

- Arreo: Es cuando el capataz deja los semovientes por fuera de los límites de la finca y los delincuentes aprovechan esa situación para espolear uno o varios animales y llevárselos. - Carneo: Conocido como pelaje, es la retención del ganado para extraer solo carne, luego de su sacrificio.

- Robo masivo: Es el delito cuando los delincuentes se llevan todo el ganado de la finca en un tipo de transporte en el menor tiempo posible [28].

\section{Metodología}

La siguiente investigación tiene un enfoque cualitativo, el cual se basa en la recolección de información a través de 
diferentes medios tales como: medios electrónicos, repositorios virtuales, periódicos, entre otros. Con la herramienta del internet se logró conseguir datos acerca de los procesos logísticos del sector ganadero y la seguridad de la cadena de suministro.

Se consideró a la Asociación Nacional de Ganaderos como la autoridad más relevante, ya que este gremio participa, unifica y representa a alrededor de 7000 ganaderos, que van desde pequeños, medianos y grandes. Un objetivo del gremio es la defensa de los intereses comunes inherentes a sus actividades. Se dividen en 9 capítulos, uno por cada provincia. El Capítulo de Chiriquí, es uno de los capítulos con mayor índice de casos denunciados sobre el hurto pecuario en el país. Por este motivo son la población meta más interesante a estudiar su nivel de madurez de riesgo.

El Capítulo de Chiriquí consta de 96 productores agremiados, los cuales se consideraron como la población a estudiar.

Entre las técnicas utilizadas para la realización de este trabajo podemos mencionar los siguientes: Revisión documental, que ayudó a analizar la situación, el problema y ejecutar posibles soluciones que mitiguen el problema de hurto pecuario. Otra de las técnicas para la debida recolección de datos fue a través de encuestas a los productores ganaderos inscritos y activos en el Capítulo de Chiriquí, al día 28 de febrero de 2019, quienes comentaron su preocupación de la situación planteada en este artículo. Dicha lista se obtuvo por parte de la propia ANAGAN, y posteriormente, se contactó con los productores / ganaderos interesados en participar en el estudio.

Se utilizaron los números telefónicos referenciados en la lista oficial en la ANAGAN, para poder hacer citas de entrevistas con los propios productores / ganaderos inscritos oficialmente en el Capítulo Chiriquí.

Esta población de encuestados la componen productores de todo tipo de escolaridad y nivel social; dado lo mencionado, y para facilidad del entendimiento de las preguntas dadas en el cuestionario, se realizaron las mismas en forma personal por parte del investigador. Con la presencia del investigador se pudo aclarar cualquier duda en la interpretación que se tuviera de cada cuestionamiento.

\subsection{Métodos de muestreo}

La muestra piloto se tomó por conveniencia siendo evaluados cuatro productores ganaderos. Luego de aplicada la prueba piloto, se detalló que el promedio del nivel de gestión de riesgo resultó en un $95 \%$ por lo que se designa un valor $\bar{x}=0.95$ y una desviación estándar de 0.318 .

Para sacar el tamaño de la muestra se utilizó la siguiente fórmula estadística sobre nivel de confiabilidad.

$$
n=\frac{z^{2} \times \sigma^{2} \times \mathrm{N}}{e^{2} \times(N-1)+z^{2} \times \sigma^{2}}
$$

En donde:

$\mathrm{N}=$ Población

$\mathrm{n}=$ Muestra

$\mathrm{z}=$ Nivel de confiabilidad

e $=$ Nivel de error aceptado para la estimación de la media muestral

$\sigma=$ Desviación estándar de la muestra

En el presente proyecto, basados en la prueba piloto, se determina el tamaño de la muestra utilizando los siguientes valores para cada variable:

$\mathrm{z}=1.96$ (Nivel de confiabilidad de 95\%)

$\mathrm{N}=96$ productores ganaderos inscritos al ANAGAN Capítulo de Chiriquí

$\mathrm{e}=0.10$

$$
\mathrm{n}=28 \text { ganaderos }
$$

\subsection{Encuestas}

Para la confección del análisis sobre la situación que se encuentra el sector ganadero en la provincia de Chiriquí, se realizó este tipo de encuesta.

\subsubsection{Modelo de madurez de riesgos}

Este artículo expresa el modelo de madurez de riesgo en el proceso de gestión de riesgo basado en la Norma ISO 31000:2009. En consecuencia, la medición de madurez de riesgo está enfocada en nivel de implementación.

Se utilizó una encuesta como herramienta para medir el nivel de madurez de riesgo (RMM). El instrumento fue diseñado según los parámetros de la Norma ISO 31000:2019, el cual fue desarrollado por el grupo de investigación de Logística y Cadena de Suministro (LOYCS) dirigido por el Dr. Práxedes Torres.

Este instrumento se ha utilizado en tres investigaciones: en una primera investigación que midió El Nivel de Madurez de Riesgo en la Administración de Riesgo de los Aeropuertos del Área Metropolitana de Panamá [29]; el segundo fue aplicado en la investigación de la Medición del Grado de Madurez de la Gestión del Riesgo en las Zonas Económicas Especiales [30] y en esta investigación que también midió el Nivel de Madurez de Riesgo, en el Sector Productor Ganadero [31].

Las empresas que tienen un proceso formalizado de gestión de riesgo para tipos particulares de riesgo, o de situaciones de riesgo, deben tener como práctica la revisión y evaluación de los atributos de una gestión de riesgo optimizada. Basados en la Norma ISO 31000, deben de ser los siguientes atributos: Mejora Continua; Responsabilidad completa de los riesgos; Aplicación de la Gestión del Riesgo en todas las decisiones; Comunicación Continua; e Integración completa en la estructura de gobierno de la organización [32]. 
La herramienta se simplificó en 14 preguntas abiertas que resumen los puntos de la Norma ISO 31000:2009 y de los atributos mencionados. En la tabla 3 se muestra la encuesta utilizada:

Tabla 3. Encuesta de grado de madurez [31]

\begin{tabular}{|c|c|c|c|c|c|c|}
\hline N. ${ }^{\circ}$ & Indicador de éxito & 1 & 2 & 3 & 4 & 5 \\
\hline 1 & $\begin{array}{l}\text { El desempeño de la organización e } \\
\text { individual se mide con indicadores } \\
\text { tangibles en forma anual. }\end{array}$ & & & & & \\
\hline 2 & $\begin{array}{l}\text { El desempeño de la organización se } \\
\text { publica y comunica. }\end{array}$ & & & & & \\
\hline 3 & $\begin{array}{l}\text { Para cada año nuevo se revisan los } \\
\text { procesos. }\end{array}$ & & & & & \\
\hline 4 & $\begin{array}{l}\text { La evaluación del desempeño de la } \\
\text { gestión de riesgo es parte de la } \\
\text { evaluación de desempeño en toda } \\
\text { la organización. }\end{array}$ & & & & & \\
\hline 5 & $\begin{array}{l}\text { El personal tiene conciencia de los } \\
\text { riesgos, controles y de las tareas de } \\
\text { las que son responsables. }\end{array}$ & & & & & \\
\hline 6 & $\begin{array}{l}\text { Se encuentran registradas, en algún } \\
\text { documento, las responsabilidades } \\
\text { individuales en la gestión de } \\
\text { riesgos. }\end{array}$ & & & & & \\
\hline 7 & $\begin{array}{l}\text { La inducción de colaboradores } \\
\text { incluye la definición de funciones, } \\
\text { rendimiento de cuentas y } \\
\text { responsabilidades de la gestión de } \\
\text { riesgos. }\end{array}$ & & & & & \\
\hline 8 & $\begin{array}{l}\text { Los responsables de gestionar } \\
\text { riesgos cuentan con los recursos } \\
\text { adecuados para su función. }\end{array}$ & & & & & \\
\hline 9 & $\begin{array}{l}\text { Hay evidencias (e.g. minutas) en la } \\
\text { que se discuten los temas de riesgos } \\
\text { en las decisiones. }\end{array}$ & & & & & \\
\hline 10 & $\begin{array}{l}\text { Las decisiones (e.g. inversión) } \\
\text { consideran la gestión del riesgo. }\end{array}$ & & & & & \\
\hline 11 & $\begin{array}{l}\text { Se hacen informes detallados y } \\
\text { frecuentes del desempeño de la } \\
\text { gestión de riesgo a los interesados } \\
\text { (internos y externos). }\end{array}$ & & & & & \\
\hline 12 & $\begin{array}{l}\text { Se hacen informes detallados y } \\
\text { frecuentes de los riesgos y su } \\
\text { tratamiento a los interesados } \\
\text { (internos y externos). }\end{array}$ & & & & & \\
\hline 13 & $\begin{array}{l}\text { La probabilidad de que ocurran } \\
\text { riesgos es considerada en la toma } \\
\text { de decisiones. }\end{array}$ & & & & & \\
\hline 14 & $\begin{array}{l}\text { En las acciones y declaraciones de } \\
\text { la administración se habla de los } \\
\text { riesgos y sus efectos. }\end{array}$ & & & & & \\
\hline
\end{tabular}

Posterior a esto, en la tabla 4 se detallan los atributos correspondientes a las preguntas de la encuesta (indicadores de éxito mencionados en la tabla 3) clasificándolas en: Mejora continua, responsabilidad completa de los riesgos, Aplicación de la gestión del riesgo en todas las decisiones, comunicación continua, integración completa en la estructura de gobierno de la organización.

Tabla 4. Atributos de indicadores de éxito [31]

\begin{tabular}{|c|c|c|}
\hline Atributos & Características & N. ${ }^{\circ}$ \\
\hline \multirow{5}{*}{ Mejora continua } & \multirow{2}{*}{$\begin{array}{l}\text { Se cuenta con metas de } \\
\text { desempeño organizacional }\end{array}$} & 1 \\
\hline & & 2 \\
\hline & \multirow{3}{*}{$\begin{array}{l}\text { Se realizan mediciones, } \\
\text { revisiones y modificaciones de } \\
\text { los procesos, los recursos, la } \\
\text { capacidad y las habilidades }\end{array}$} & 3 \\
\hline & & \\
\hline & & 4 \\
\hline \multirow{3}{*}{$\begin{array}{l}\text { Responsabilidad } \\
\text { completa de los } \\
\text { riesgos }\end{array}$} & \multirow{3}{*}{$\begin{array}{l}\text { La responsabilidad de } \\
\text { identificar, controlar y tratar los } \\
\text { riesgos está definida y aceptada }\end{array}$} & 5 \\
\hline & & 6 \\
\hline & & 7 \\
\hline \multirow{3}{*}{$\begin{array}{l}\text { Aplicación de la } \\
\text { gestión del } \\
\text { riesgo en todas } \\
\text { las decisiones }\end{array}$} & \multirow{3}{*}{$\begin{array}{l}\text { Los responsables de gestionar } \\
\text { riesgos cuentan con los recursos } \\
\text { adecuados para su función }\end{array}$} & 8 \\
\hline & & 9 \\
\hline & & 10 \\
\hline \multirow{2}{*}{$\begin{array}{l}\text { Comunicación } \\
\text { continua }\end{array}$} & \multirow{2}{*}{$\begin{array}{l}\text { Hay comunicación continua } \\
\text { entre la organización y las partes } \\
\text { interesadas sobre la gestión de } \\
\text { riesgo }\end{array}$} & 11 \\
\hline & & 12 \\
\hline \multirow[b]{2}{*}{$\begin{array}{l}\text { Integración } \\
\text { completa en la } \\
\text { estructura de } \\
\text { gobierno de la } \\
\text { organización } \\
\end{array}$} & \multirow{2}{*}{$\begin{array}{l}\text { Se consideran los riesgos y sus } \\
\text { efectos en el alcance de los } \\
\text { objetivos de la organización }\end{array}$} & 13 \\
\hline & & 14 \\
\hline
\end{tabular}

En la tabla 5 se presenta el rango de puntaje a utilizar indicando el grado de madurez junto a su descripción.

Tabla 5. Puntaje para la evaluación del grado de madurez [31]

\begin{tabular}{|c|c|l|}
\hline $\begin{array}{c}\text { Grado de } \\
\text { Madurez }\end{array}$ & $\begin{array}{c}\text { Rango de } \\
\text { Puntaje }\end{array}$ & \multicolumn{1}{c|}{ Descripción } \\
\hline Bajo (L) & $1.00-1.92$ & $\begin{array}{l}\text { Existe un conocimiento } \\
\text { nulo o mínimo en la } \\
\text { organización acerca de la } \\
\text { necesidad de administrar } \\
\text { los riesgos y no hay } \\
\text { procesos puestos en } \\
\text { marcha. }\end{array}$ \\
\hline $\begin{array}{c}\text { Inconsistente } \\
\text { (I) }\end{array}$ & $1.93-2.92$ & $\begin{array}{l}\text { Existe un conocimiento } \\
\text { acerca de la importancia } \\
\text { de administrar los riesgos } \\
\text { y solo algunas áreas de la } \\
\text { organización tienen los } \\
\text { procesos puestos en } \\
\text { marcha. }\end{array}$ \\
\hline
\end{tabular}




\begin{tabular}{|c|c|c|}
\hline $\begin{array}{l}\text { Grado de } \\
\text { madurez }\end{array}$ & $\begin{array}{l}\text { Rango de } \\
\text { puntaje }\end{array}$ & Descripción \\
\hline $\begin{array}{l}\text { Medianament } \\
\text { e consistente } \\
\text { (M) }\end{array}$ & $2.93-3.92$ & $\begin{array}{l}\text { Existe un conocimiento } \\
\text { moderado de la } \\
\text { importancia de la } \\
\text { administración de } \\
\text { riesgos, pero aún existen } \\
\text { áreas en las que se debe } \\
\text { mejorar. }\end{array}$ \\
\hline $\begin{array}{c}\text { Consistente } \\
\text { (C) }\end{array}$ & $3.93-4.92$ & $\begin{array}{l}\text { Existe un claro } \\
\text { compromiso } \\
\text { organizacional y hay } \\
\text { procesos comunes } \\
\text { utilizados en toda la } \\
\text { organización. Sin } \\
\text { embargo, aún existen } \\
\text { áreas en las que se debe } \\
\text { mejorar. }\end{array}$ \\
\hline $\begin{array}{c}\text { Totalmente } \\
\text { alcanzado }(F)\end{array}$ & $4.93-5.00$ & $\begin{array}{l}\text { Existe un claro } \\
\text { compromiso } \\
\text { organizacional y hay } \\
\text { procesos comunes } \\
\text { utilizados en toda la } \\
\text { organización. } \\
\text { Monitorean } \\
\text { rutinariamente su } \\
\text { desempeño para verificar } \\
\text { su efectividad y hacen } \\
\text { mejoras si es necesario. }\end{array}$ \\
\hline
\end{tabular}

\section{Resultados}

El nivel alcanzado fue de un nivel de madurez de la administración de riesgos medianamente consistente (2.96).

Se muestra en la tabla 6 el nivel de cumplimiento de madurez por atributo para el sector ganadero con su porcentaje de alcance.

Tabla 6. Nivel de madurez de la gestión de riesgo

\begin{tabular}{|l|c|c|}
\hline \multicolumn{1}{|c|}{ Atributo } & Puntuación & $\begin{array}{c}\text { Porcentaje de } \\
\text { alcance }\end{array}$ \\
\hline Mejora Continua & 2.71 & $54 \%$ \\
\hline $\begin{array}{l}\text { Responsabilidad } \\
\text { completa de los riesgos }\end{array}$ & 3.10 & $62 \%$ \\
\hline $\begin{array}{l}\text { Aplicación de la } \\
\text { gestión del riesgo en } \\
\text { todas las decisiones }\end{array}$ & 2.38 & $48 \%$ \\
\hline Comunicación continua & 2.71 & $54 \%$ \\
\hline $\begin{array}{l}\text { Integración completa } \\
\text { en la estructura de } \\
\text { gobierno de la } \\
\text { organización }\end{array}$ & 3.93 & $79 \%$ \\
\hline \multicolumn{1}{|c|}{ Total general } & 2.96 & $59 \%$ \\
\hline
\end{tabular}

En general las características que se tienen en el sector ganadero son:

- Mejora continua: Nivel 2 - Inconsistente

Existe un conocimiento acerca de la importancia de administrar los riesgos y solo algunas áreas de la organización tienen los procesos puestos en marcha.

- Responsabilidad completa de los riesgos: Nivel 3 Medianamente consistente

Existe un conocimiento moderado de la importancia de la administración de riesgos, pero aún existen áreas que no son tratadas.

- Aplicación de la gestión del riesgo en todas las decisiones: Nivel 2 - Inconsistente

Existe un conocimiento acerca de la importancia de administrar los riesgos y solo algunas áreas de la organización tienen los procesos puestos en marchas.

- Comunicación continua: Nivel 2 - Inconsistente

Al igual que las características mencionadas como la Mejora continua y la aplicación de la gestión del riesgo en todas las decisiones, existe un conocimiento acerca de la importancia de administrar los riesgos y solo algunas áreas de la organización tienen los procesos puestos en marchas.

- Integración completa en la estructura de gobierno de la organización: Nivel 4 - Consistente

Existe un claro compromiso organizacional y hay procesos comunes utilizados en toda la organización. Sin embargo, aún existen áreas en las que se debe mejorar.

En la tabla 7 se presenta los resultados generales para el sector ganadero.

Tabla 7. Resultados generales para el sector ganadero encuestado

\begin{tabular}{|c|c|c|}
\hline $\begin{array}{c}\text { Productores } \\
\text { ganaderos }\end{array}$ & $\begin{array}{c}\text { Puntuación } \\
\text { total }\end{array}$ & Grado de madurez \\
\hline 1 & 2.30 & Inconsistente \\
\hline 2 & 3.20 & $\begin{array}{c}\text { Medianamente } \\
\text { consistente }\end{array}$ \\
\hline 3 & 3.90 & $\begin{array}{c}\text { Medianamente } \\
\text { consistente }\end{array}$ \\
\hline 4 & 3.40 & $\begin{array}{c}\text { Medianamente } \\
\text { consistente }\end{array}$ \\
\hline 5 & 2.70 & \begin{tabular}{c} 
Inconsistente \\
\hline 6
\end{tabular} \\
\hline 7 & 3.60 & $\begin{array}{c}\text { Medianamente } \\
\text { consistente }\end{array}$ \\
\hline 8 & 2.40 & Inconsistente \\
\hline 9 & 2.95 & Inconsistente \\
\hline 10 & 2.20 & $\begin{array}{c}\text { Medianamente } \\
\text { consistente }\end{array}$ \\
\hline 11 & 3.00 & Inconsistente \\
\hline & & $\begin{array}{c}\text { Medianamente } \\
\text { consistente }\end{array}$ \\
\hline
\end{tabular}




\begin{tabular}{|c|c|c|}
\hline $\begin{array}{l}\text { Productores } \\
\text { ganaderos }\end{array}$ & $\begin{array}{c}\text { Puntuación } \\
\text { total }\end{array}$ & Grado de madurez \\
\hline 12 & 3.95 & $\begin{array}{l}\text { Medianamente } \\
\text { consistente }\end{array}$ \\
\hline 13 & 3.45 & $\begin{array}{l}\text { Medianamente } \\
\text { consistente }\end{array}$ \\
\hline 14 & 2.60 & Inconsistente \\
\hline 15 & 2.55 & Inconsistente \\
\hline 16 & 2.75 & Inconsistente \\
\hline 17 & 3.05 & $\begin{array}{l}\text { Medianamente } \\
\text { consistente }\end{array}$ \\
\hline 18 & 3.30 & $\begin{array}{l}\text { Medianamente } \\
\text { consistente }\end{array}$ \\
\hline 19 & 2.75 & Inconsistente \\
\hline 20 & 3.80 & $\begin{array}{l}\text { Medianamente } \\
\text { consistente }\end{array}$ \\
\hline 21 & 2.60 & Inconsistente \\
\hline 22 & 3.30 & $\begin{array}{l}\text { Medianamente } \\
\text { consistente }\end{array}$ \\
\hline 23 & 2.05 & Inconsistente \\
\hline 24 & 3.15 & $\begin{array}{l}\text { Medianamente } \\
\text { consistente }\end{array}$ \\
\hline 25 & 2.60 & Inconsistente \\
\hline 26 & 2.75 & Inconsistente \\
\hline 27 & 4.35 & Consistente \\
\hline 28 & 2.00 & Inconsistente \\
\hline Promedio & 2.96 & \\
\hline
\end{tabular}

Dentro de los elementos del sistema de gestión de seguridad de la Norma ISO 31000 se cuenta con la preparación ante emergencias. Esto conlleva a planes y procedimientos para la identificación, respuestas y gestión de los potenciales incidentes a la seguridad y las acciones de emergencias [31].

La mayoría de los productores ganaderos encuestados no cuentan con planes de emergencias (68\%), por lo que es fundamental establecer, implementar, mantener planes y procedimientos apropiados para evitar y mitigar los incidentes de seguridad y situaciones de emergencias que pudiesen ocurrir en un momento determinado [31].

\section{Conclusiones}

- Dado que los productores tienen un nivel de madurez medianamente consistente, deben tener un claro compromiso organizacional y contar con procesos comunes utilizados en toda la organización. Es necesario contar con el monitoreo rutinariamente del desempeño para verificar su efectividad y hacer mejoras si es necesario.

- Se debe realizar planes y procedimientos para la identificación, respuestas y gestión de los potenciales incidentes a la seguridad y las acciones de emergencias, ya que la mayoría de los productores ganaderos no cuentan con planes de emergencias que estipula la norma ISO 31000, por lo que es fundamental establecer, implementar, mantener planes y procedimientos apropiados para evitar y mitigar los incidentes de seguridad y situaciones de emergencias que pudiesen ocurrir en un momento determinado.

- Este sector reconoce que, si no se toman medidas para considerar soluciones o mejoras, y a su vez las comprobaciones periódicas (control y/o auditoría), hay una alta vulnerabilidad de sufrir incidentes que podría conllevar a pérdidas económicas dentro de la empresa.

- El impacto que tiene en la logística el rompimiento de la cadena de suministro al tener problemas primordialmente en temas de "security" entre los productores ganaderos genera importantes pérdidas económicas, ya que se interrumpe con la continuidad de los procesos de transformación de la materia prima hasta su consumidor final.

\section{AGRADECIMIENTO}

A la Asociación Nacional de Ganaderos de Panamá Capítulo Chiriquí por permitirnos recopilar información, a los productores ganaderos que aceptaron ser entrevistados.

\section{REFERENCIAS}

[1] International Dynamic Advisors, «Seguridad para la Cadena de Suministro,» 27 Abril 2016. [En línea]. Available: http://www.intedya.com/internacional/166/consultoriaestandares-nacionales-para-la-gestion-de-la-seguridad-de-lacadena-de-suministro.html\#submenuhome.

[2] Instituto Uruguayo de Normas Técnicas, «Historia/ Antecedentes,» 4 enero 2019. [En línea]. Available: https://www.unit.org.uy/normalizacion/sistema/28000/.

[3] M. Romero, CONFERENCIA - ISO 28000 Enfoque de gestión de riesgos para la cadena de suministro, 2017.

[4] Redacción Digital El Siglo, «Funcionarios de la Autoridad Nacional de Aduanas en paro de 24 horas,» 6 diciembre 2018.

[5] I. Franco, «Paralización en Aduanas podría prorrogarse,» Mi Diario, 9 diciembre 2018.

[6] G. León Barría, «Médicos Veterinarios convocan paro de brazos caídos a partir de este lunes,» 09 diciembre 2018.

[7] C. Castillo, «Sector agrícola de Panamá está abocado a la crisis,» Panamá América, 30 julio 2018.

[8] Redacción Digital La Estrella, «El MP recibió 651 denuncias por hurto pecuario,» 08 septiembre 2017.

[9] Ministerio Público, «Cinco personas condenadas por hurto pecuario agravado,» 10 enero 2019. [En línea]. Available: http://ministeriopublico.gob.pa/cinco-personas-condenadaspor-hurto-pecuario-agravado/. 
[10] Contraloría General de la República de Panamá, «Explotaciones y existencia de ganado vacuno en la República, por clase, según provincia, comarca indígena, distrito y actividad del hato,» Panamá, 2011.

[11] V. M. Nieto Galindo y N. Ramírez, «Cadena productiva de Carnes y Productos Cárnicos. Estructura, Comercio Internacional y Protección,» Colombia, 2018.

[12] LTX Team, «Protecting Your Supply Chain: Security and Risk Management,» 29 julio 2018. [En línea]. Available: http://ltxsolutions.com/protecting-supply-chain-security-riskmanagement/.

[13] G. P. Salas, «Recursos Naturales e Infraestructura,» Marzo 2013. [En línea]. Available: https://repositorio.cepal.org/bitstream/handle/11362/6365/LC L3604_es.pdf?sequence $=1$.

[14] CEPAL, «Seguridad de la cadena logística y su incidencia en la competitividad de Mesoamérica,» Agosto 2011. [En línea]. Available: https://repositorio.cepal.org/bitstream/handle/11362/36084/1/ FAL-300-WEB_es.pdf.

[15] DNV GL, «ISO 28000 - Sistema de gestión de Seguridad para la Cadena de Suministro,» 04 enero 2019. [En línea]. Available: https://www.dnvgl.es/services/iso-28000-sistemade-gestion-de-seguridad-para-la-cadena-de-suministro-4344.

[16] M. Romero, «Norma ISO 28000,» Bogotá, 2019.

[17] Instituto Uruguayo de Normas Técnicas, «UNIT-ISO 28000 Gestión de la seguridad para la cadena de suministro,» 4 enero 2019. [En línea]. Available: https://www.unit.org.uy/normalizacion/sistema/28000/.

[18] International Organization for Standardization, «ISO 28000:2007 Specification for security management systems for the supply chain,» 4 enero 2019. [En línea]. Available: https://www.iso.org/standard/44641.html.

[19] F. C. Pauw, «Seguridad en la cadena de suministro,» 22 abril 2019. [En línea]. Available: http://www.seguritecnia.es/seguridad-aplicada/comercio-ydistribucion/seguridad-en-la-cadena-de-suministro.

[20] Icontec Internacional, «ISO 28000 Sistemas de gestión de la seguridad en la cadena de suministro,» 10 abril 2019. [En línea]. Available: http://medios.icontec.org/documentos/Memorias\%20Charla\% 20Virtual\%2010\%20de\%20Abril.pdf.

[21] Á. Olleros, «Diferencias entre Seguridad, Safety y Security,» 13 marzo 2017. [En línea]. Available: https://www.angelolleros.com/diferencias-seguridad-safetysecurity/.

[22] F. Davara, «Security \& Safety; enfoques diferentes de la seguridad,» 2017. [En línea]. Available: http://fernandodavara.com/security-enfoques-diferentes-laseguridad/.

[23] E. Albrechtsen, «Security vs safety,» Agosto 2003. [En línea]. Available: http://www.iot.ntnu.no/users/albrecht/rapporter/notat\%20safet y\%20v\%20security.pdf.

[24] Labour Prevención de Riesgos S.L., «Riesgos y medidas preventivas en ganadería y agricultura,» [En línea]. Available: https://www.insst.es/ganaderia. [Último acceso: 20 agosto 2019].

[25] A. C. Liendo, «Modelos de Madurez,» 2016. [En línea]. Available: https://projectools.wordpress.com/modelos-demadurez-en-gestion-de-proyectos/.

[26] RIMS RMM Community, «The RIMS Risks Maturity Model,» 4 octubre 2019. [En línea]. Available: https://www.riskmaturitymodel.org/.

[27] K. Aguilar Morán, «Incidencias de la norma ISO 28000 en los procesos logístico de las empresas exportadoras de camarón de la zona 5 ,» Guayaquil, 2018.

[28] A. Alarcón, «Sistema de monitorización y control de ganado vacuno por medio de tecnología inalámbrica para prevención de abigeato,» Ambato, 2018.

[29] L. A. Cedeño Acevedo y J. B. Ortega Aguilar, «Evaluación del grado de madurez de la administración de riesgos en los aeropuertos del área metropolitana de Panamá,» Universidad Tecnológica de Panamá, Panamá, 2016.

[30] Y. Gil y Y. Espinoza, «Evaluación de la gestión de riesgo en las principales zonas económicas especiales de Panamá,» Universidad Tecnológica de Panamá, Panamá, 2017.

[31] E. R. Saldaña Patiño, «Evaluación de la gestión de seguridad en la cadena de suministro en el sector ganadero de Panamá,» Universidad Tecnológica de Panamá, Panamá, 2019.

[32] I. Casares San José-Martí, «Gestión del riesgo optimizada,» 2014. [En línea]. Available: https://fundacioninade.org/sites/inade.org/files/web_2014__gestion_del_riesgo_optimizada.pdf. [Último acceso: 21 agosto 2020]. 